\title{
COMPORTAMENTO AGRONÔMICO DE LINHAGENS DE TRIGO NO ESTADO DE SÃO PAULO ${ }^{(1)}$
}

\author{
CARLOS EDUARDO DE OLIVEIRA CAMARGO ${ }^{(2,6)}$; JOÃO CARLOS \\ FELÍCIO $^{(2)}$; ANTONIO WILSON PENTEADO FERREIRA FILHO ${ }^{(2)}$; \\ BENEDITO DE CAMARGO BARROS ${ }^{(3,6)}$; JOSÉ CARLOS VILA NOVA ALVES \\ PEREIRA $^{(4)}$; ARMANDO PETTINELLI JUNIOR ${ }^{(5)}$
}

\begin{abstract}
RESUMO
Compararam-se 18 linhagens de trigo e os cultivares IAC-24 e IAC-289 em seis ensaios instalados em condições de irrigação por aspersão e de sequeiro. Foram analisadas a produção de grãos, a resistência a doenças e outras características agronômicas Em condições de laboratório estudou-se a tolerância ao alumínio em soluções nutritivas. A linhagem L9 e o cultivar IAC-289 destacaram-se quanto à produção de grãos, considerando a média dos seis experimentos. A linhagem L8 exibiu porte baixo associado à resistência ao acamamento e ciclo precoce da emergência à maturação. Em relação à ferrugem-da-folha, as linhagens L1, L17 e L20 revelaram características de resistência. Todos os genótipos mostraram-se sensíveis aos agentes causais das manchas foliares e de oídio. A linhagem L7 apresentou espigas mais compridas e maior número de espiguetas por espiga; o cultivar IAC-289, maior número de grãos por espiga; a linhagem L17, maior número de grãos por espigueta; e a linhagem L11, grãos mais pesados. Todos os genótipos avaliados mostraram-se tolerantes à toxicidade de $\mathrm{Al}^{3+}$, com exceção do cultivar-controle Anahuac e das linhagens L15 e L20, que exibiram elevada sensibilidade.
\end{abstract}

Palavras-chave: trigo, características agronômicas, resistência a doenças, tolerância ao alumínio.

\section{ABSTRACT \\ AGRONOMIC PERFORMANCE OF WHEAT HYBRID LINES IN THE STATE OF SÃO PAULO, BRAZIL}

Eighteen wheat lines and the check cultivars IAC-24 and IAC-289 were evaluated in six trials carried out at different locations under dryland and sprinkler irrigation conditions, for grain yield, agronomic characteristics and disease resistance. In laboratory conditions, the germplasms were evaluated for their Al toxicity tolerance in nutrient solutions. The line L9 and the cultivar IAC-289 were superior in grain yield, considering the average of the six trials. The line L8 exhibited short stature associated to lodging resistance and early maturity. In relation to leaf rust the lines L1, L17 and L20 were resistant. All genotypes were susceptible to the causal agents of leaf spot and mildew. The line L7, showed long heads with the highest number of spikelets; IAC-289 and L17, the largest number of grain per head and per spikelet, respectively; and L11, the heaviest grains. All evaluated genotypes

$\left({ }^{1}\right)$ Trabalho parcialmente financiado pela FAPESP. Recebido para publicação em 10 de novembro de 1999 e aceito em 7 de fevereiro de 2000.

$\left(\begin{array}{l}2 \\ 3\end{array}\right)$ Instituto Agronômico (IAC), Centro de Plantas Graníferas, Caixa Postal 28, 13001-970 Campinas (SP).

$\left({ }^{3}\right)$ Centro Experimental do Instituto Biológico (IB), Caixa Postal 70, 13001-970 Campinas (SP).

$\left({ }^{4}\right)$ Núcleo de Agronomia da Alta Mogiana, IAC, Caixa Postal 271, 14001-970 Ribeirão Preto (SP).

$(5)$ Estação Experimental de Agronomia de Tatuí, IAC, Caixa Postal 33, 18270-000 Tatuí (SP).

(6) Com bolsa de produtividade em pesquisa do CNPq. 
were tolerant to $\mathrm{Al}^{3+}$ toxicity, excepting the control cultivar Anahuac and the lines $\mathrm{L} 15$ and L20, which exhibited high sensibility.

Key words: wheat, agronomic characteristics, disease resistance, aluminum tolerance.

\section{INTRODUÇÃO}

Os trabalhos de obtenção de novos cultivares de trigo devem considerar, para o Estado de São Paulo, os seguintes aspectos: maior produtividade, porte semi-anão, palha forte (resistência ao acamamento), maior fertilidade da espiga, maior perfilhamento, precocidade (ciclo da emergência à maturação de 100 a 120 dias para favorecer a rotação com outras culturas), resistência à degrana, resposta à adubação, índice de colheita mais alto ( relação entre a massa dos grãos e a massa total da parte aérea da planta), adaptação ampla, resistência a doenças (ferrugens, oídio e helmintosporiose), tolerância a níveis tóxicos de alumínio e manganês e melhor qualidade nutritiva e tecnológica. Obtido o cultivar, faz-se necessário um programa eficiente de multiplicação de sementes visando à agilização do fornecimento do novo material genético aos agricultores (CAMARGO, 1987; 1993; CAMARGO et al., 1996; KoHLI et al., 1994).

O Instituto Agronômico vem introduzindo, anualmente, coleções de variedades e populações híbridas, em geração $F_{2}$, do Centro Internacional de Melhoramento de Milho e Trigo (CIMMYT) (México) e da Universidade Estadual de Oregon (EUA), visando detectar fontes de resistência à ferrugem-do-colmo e à ferrugem-da-folha, para serem tais variedades e populações híbridas utilizadas no programa de melhoramento, uma vez que a maioria das variedades atualmente recomendadas para as diferentes regiões tritícolas paulistas é suscetível a, pelo menos, uma raça dessas ferrugens, necessitando, pois, controle com fungicida em anos favoráveis ao seu desenvolvimento (CAMARGO et al., 1996).

Variedades de origem mexicana foram recomendadas a partir de 1970, após avaliação em rede de experimentação regional, pelo alto potencial produtivo aliado ao porte baixo, resistência ao acamamento e às ferrugens, somente para solos corrigidos, em vista da elevada sensibilidade dessas variedades ao $\mathrm{Al}^{3+}$, presente no solo. Para tais condições foram lançados, mais recentemente, os cultivares Anahuac, IAC-287, IAC-289 e IAC-350 (Felício et al., 1994a,b; 1996; CAMARgo et al., 1996).

O programa de melhoramento genético do trigo no IAC tem procurado, através de cruzamentos entre variedades nacionais e linhagens semi-anãs de origem mexicana, desenvolver variedades de porte semi-anão de alto potencial produtivo e com tolerância à toxicidade de $\mathrm{Al}^{3+}$. As variedades IAC-24, IAC-
60 e IAC-227 e, mais recentemente, IAC-120 e IAC-231 foram lançadas, sendo as primeiras a apresentarem tais características (Felício et al., 1994a,b; 1991; 1988; CAMARGo et al., 1996). A variedade IAC-24 tem mostrado excelentes qualidades tecnológicas para panificação.

Este trabalho objetiva avaliar, em condição de sequeiro e de irrigação, o comportamento de 18 linhagens de trigo, em comparação com 'IAC-24' e 'IAC-289', visando à escolha das mais promissoras, tanto para multiplicação e posterior lançamento aos triticultores paulistas, quanto para utilizá-las como fontes genéticas de interesse no programa de melhoramento do Instituto Agronômico.

\section{MATERIAL E MÉTODOS}

Incluíram-se nos ensaios 14 genótipos (1 a 9, 11, 12, 14,17 e 18), provenientes de cruzamentos entre linhagens selecionadas em ensaios introduzidos do Centro Internacional de Melhoramento de Milho e Trigo (CIMMYT) (México) e variedades nacionais (IAC) recomendadas para cultivo no Estado de São Paulo -, 4 linhagens mexicanas oriundas do CIMMYT $(13,15$, 16 e 20) e os cultivares-controle IAC-24 e IAC-289.

Empregou-se o delineamento estatístico de blocos ao acaso, com quatro repetições por local. Cada ensaio constituiu-se de 80 parcelas, cada uma formada por seis linhas de $3 \mathrm{~m}$ de comprimento, espaçadas de 0,20 m. Deixou-se uma separação lateral de 0,60 m entre as parcelas, semeando-se 80 sementes viáveis por metro linear de sulco, com uma área útil de colheita de $3,6 \mathrm{~m}^{2}$.

Em 1996 e 1997 realizaram-se três ensaios, nos seguintes locais: Estação Experimental de Agronomia de Tatuí, Núcleo de Agronomia da Alta Mogiana, em Ribeirão Preto e Fazenda Santa Lúcia, município de Cruzália.

Retiraram-se amostras compostas dos solos dos locais estudados, nas profundidades de 0-20, 20-40 e 40-60 cm, encontrando-se, no quadro 1 , os resultados das análises. Utilizou-se irrigação pelo método de aspersão somente nos ensaios de Ribeirão Preto e Tatuí.

A relação e a genealogia dos 20 genótipos avaliados são apresentadas no quadro 2.

Os genótipos foram comparados quanto à resistência à ferrugem-da-folha (Puccinia triticina), mancha foliar (Bipolaris sorokiniana) e oídio (Blumeria 
Quadro 1. Análises das amostras dos solos ${ }^{(1)}$ nas profundidades 0-20, 20-40 e 40-60, dos locais onde foram instalados os experimentos em 1996 e 1997

\begin{tabular}{|c|c|c|c|c|c|c|c|c|c|c|c|}
\hline Local & Prof. & $\mathrm{P}$ & M.O & $\mathrm{pH}-\mathrm{CaCl}_{2}$ & $\mathrm{~K}$ & $\mathrm{Ca}$ & $\mathrm{Mg}$ & $\mathrm{H}+\mathrm{Al}$ & $\mathrm{S}$ & $\mathrm{T}$ & $\mathrm{V}$ \\
\hline & $\mathrm{cm}$ & mg.dm ${ }^{3}$ & g. $\mathrm{kg}^{-1}$ & $\ldots$ & 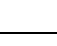 & ـ & $\mathrm{mmol}_{\mathrm{C}} \cdot \mathrm{dm}^{-3}$ & & & - & $\%$ \\
\hline \multirow[t]{3}{*}{ Cruzália } & $0-20$ & 22 & 34 & 5,2 & 3,6 & 33 & 13 & 43 & 50 & 93 & 54 \\
\hline & $20-40$ & 3 & 19 & 4,8 & 1,7 & 16 & 9 & 43 & 27 & 70 & 39 \\
\hline & $40-60$ & 6 & 13 & 4,7 & 1,3 & 14 & 7 & 43 & 22 & 65 & 34 \\
\hline \multirow[t]{3}{*}{ Ribeirão Preto } & $0-20$ & 39 & 30 & 5,2 & 2,3 & 31 & 14 & 38 & 47 & 85 & 55 \\
\hline & $20-40$ & 17 & 16 & 5,1 & 1,2 & 40 & 14 & 38 & 55 & 93 & 59 \\
\hline & $40-60$ & 17 & 19 & 5,0 & 2,9 & 40 & 15 & 43 & 58 & 101 & 57 \\
\hline \multirow[t]{3}{*}{ Tatuí } & $0-20$ & 64 & 25 & 5,3 & 6,0 & 60 & 15 & 34 & 81 & 115 & 70 \\
\hline & $20-40$ & 29 & 23 & 4,7 & 3,8 & 41 & 13 & 52 & 58 & 110 & 53 \\
\hline & $40-60$ & 7 & 19 & 4,1 & 2,5 & 21 & 9 & 99 & 33 & 132 & 25 \\
\hline
\end{tabular}

${ }^{(1)}$ Análises efetuadas pela Seção de Fertilidade do Solo e Nutrição de Plantas, IAC.

graminis f. sp.tritici), pela avaliação da severidade das doenças sob infecção natural.

A ferrugem-da-folha foi avaliada em cada parcela, através da observação nas folhas superiores das plantas nos estádios entre inicio de maturação e cera mole (estádios 11.1 e 11.2 da escala de Feekes-Large), baseando-se em escala modificada de Cobb, conforme ilustração em Menta (1978), que leva em conta a porcentagem de área afetada e o tipo de pústula, ou seja, a intensidade da doença e a reação da planta.

Para mancha foliar e oídio, tomou-se como base para avaliação uma escala de porcentagem de área foliar infectada, apresentada por MEHTA (1978).

Outras características, como o ciclo das plantas, em dias, da emergência à maturação, altura das plantas, acamamento, comprimento das espigas, número de espiguetas por espiga, número de grãos por espiga e por espigueta, massa de cem grãos e produção de grãos foram também avaliadas (CAMARGO et al. 1989, 1991; 1995a). Para a determinação do comprimento das espigas, do número de espiguetas por espiga, do número de grãos por espiga e por espigueta, e da massa de cem grãos, foram colhidas dez espigas de cada parcela, apenas nos experimentos instalados em Ribeirão Preto e Tatuí.

Os dados de produção de grãos, comprimento das espigas, número de espiguetas por espiga, número de grãos por espiga e por espigueta e massa de cem grãos de cada experimento foram submetidos à análise de variância, utilizando-se o teste $\mathrm{F}$, ao nível de $5 \%$, para detectar efeitos significativos de genótipos e repetições.

Efetuaram-se análises conjuntas da variância para produção de grãos dos experimentos de Ribeirão Preto, Cruzália e Tatuí, para detectar pelo teste $\mathrm{F}$ ao nível de 5\% a significância dos efeitos de anos, genótipos e interação genótipos $x$ anos. Realizou-se também uma análise conjunta da variância para produção de grãos dos seis experimentos, independentemente dos locais e anos onde foram instalados, para detectar pelo teste F ao nível de 5\% a significância dos efeitos de genótipos, anos, locais, as interações genótipos $\mathrm{x}$ anos, genótipos $\mathrm{x}$ locais e genótipos $\mathrm{x}$ anos $\mathrm{x}$ locais.

Realizaram-se análises conjuntas da variância para comprimento das espigas, número de espiguetas por espiga, número de grãos por espiga e por espigueta e massa de cem grãos, dos experimentos de Ribeirão Preto e Tatuí, para detectar pelo teste $\mathrm{F}$ ao nível de $5 \%$ a significância dos efeitos de experimentos, genótipos e interação genótipos x experimentos.

O teste de Tukey ao nível de 5\% foi empregado para a comparação dos genótipos nos grupos de experimentos, usando-se como estimativas do desviopadrão residual os quadrados médios da interação genótipos $\mathrm{x}$ anos ou genótipos $\mathrm{x}$ experimentos, conforme $o$ caso.

Desenvolveu-se análise conjunta da variância para altura das plantas considerando a média dessa característica em cada um dos experimentos para detectar, pelo teste $\mathrm{F}$ ao nível de $5 \%$, as significâncias dos efeitos de genótipos e experimentos. A comparação das médias dos genótipos para essa característica foi feita também pelo teste de Tukey ao nível de 5\%.

As plântulas dos 20 genótipos e dos cultivarescontrole BH-1146 (tolerante) e Anahuac (sensível) foram testadas para tolerância ao alumínio, em condição de laboratório, nas doses de 0, 2, 4, 6, 8 e $10 \mathrm{mg} . \mathrm{L}^{-1}$ de $\mathrm{Al}^{3+}$, em soluções nutritivas. O delineamento experimental empregado foi o de blocos ao acaso, com arranjo em parcelas subdivididas: as par- 
Quadro 2. Produção média de grãos dos 20 genótipos de trigo avaliados nos experimentos instalados nas Estações Experimentais de Tatuí e de Ribeirão Preto, e na Fazenda Santa Lúcia, em Cruzália, em 1996 e 1997 e resultados da análise estatistica

\begin{tabular}{|c|c|c|c|c|c|}
\hline \multicolumn{2}{|c|}{ Genótipos } & Tatuí & Cruzália & Ribeirão Preto & Média \\
\hline \multirow[t]{2}{*}{01} & H.11232 = SX/S948A1/4*BZA $/ 3 / 2 * S X / 5 /$ & & & & \\
\hline & IAC-21 & $3.564 \mathrm{~cd}$ & $1.619 \mathrm{a}-\mathrm{d}$ & $4.585 \mathrm{~cd}$ & $3.256 \mathrm{~d}-\mathrm{g}$ \\
\hline \multirow[t]{2}{*}{02} & H.11173 = KAL/BB//MON “S”/3/CNDR “S” & & & & \\
\hline & /ANA/ /CNDR “S” /MUS “S” / 4/IAC-24 & $3.840 \mathrm{a}-\mathrm{d}$ & $1.446 \mathrm{~b}-\mathrm{d}$ & $4.964 \mathrm{a}-\mathrm{d}$ & $3.417 \mathrm{~b}-\mathrm{g}$ \\
\hline 03 & H.11305 = IAS54/ALD “S” / / IAC-24 & $4.036 \mathrm{a}-\mathrm{d}$ & $1.529 \mathrm{a}-\mathrm{d}$ & $5.072 \mathrm{a}-\mathrm{d}$ & $3.546 \mathrm{~b}-\mathrm{f}$ \\
\hline 04 & H.11372 = YECORA 70/IAC-60 & $3.529 \mathrm{~cd}$ & $1.421 \mathrm{~b}-\mathrm{d}$ & $5.204 \mathrm{a}-\mathrm{d}$ & $3.384 \mathrm{c}-\mathrm{g}$ \\
\hline \multirow[t]{2}{*}{05} & H.12264 = COOK/VEE “S” / / DOVE “S”/ & & & & \\
\hline & SERI/3/IAC-24 & $3.379 \mathrm{~cd}$ & $1.228 \mathrm{~d}$ & $4.811 \mathrm{a}-\mathrm{d}$ & $3.140 \mathrm{fg}$ \\
\hline 06 & H.12480 = KAUZ "S" /IAC-24 & 3.751 a-d & $1.385 \mathrm{~b}-\mathrm{d}$ & 5.176 a-d & $3.437 \mathrm{~b}-\mathrm{g}$ \\
\hline 07 & H.12487 = PSN “S” / BOW “S" / /IAC-24 & $3.265 \mathrm{~d}$ & $1.281 \mathrm{~cd}$ & $4.472 \mathrm{~cd}$ & $3.006 \mathrm{gh}$ \\
\hline 08 & H.12261 = VEE “S” /IAC-24 & 3.974 a-d & $1.440 \mathrm{~b}-\mathrm{d}$ & $5.091 \mathrm{a}-\mathrm{d}$ & $3.502 b-f$ \\
\hline 09 & H13901 = VEE“S” / /YD“S” /PCI“S”/3/IAC24 & $4.615 \mathrm{a}$ & $2.010 \mathrm{a}$ & $5.437 \mathrm{a}-\mathrm{c}$ & $4.021 \mathrm{a}$ \\
\hline 10 & IAC-24 & $4.514 \mathrm{ab}$ & $1.691 \mathrm{a}-\mathrm{d}$ & $4.729 \mathrm{a}-\mathrm{d}$ & 3.645 a-e \\
\hline \multirow[t]{2}{*}{11} & H.13916 = CHAT “S” / / MAYA “S” / MON & & & & \\
\hline & “S”/3/IAC-24 & $4.120 \mathrm{a}-\mathrm{d}$ & $1.863 \mathrm{ab}$ & $5.603 \mathrm{ab}$ & $3.862 \mathrm{ab}$ \\
\hline 12 & H.11470 = JUN “S” /IAC-60 & $4.212 \mathrm{a}-\mathrm{c}$ & $1.837 \mathrm{ab}$ & $5.444 \mathrm{a}-\mathrm{c}$ & $3.831 \mathrm{a}-\mathrm{c}$ \\
\hline 13 & IPPO 10/CUCURPE & $4.015 \mathrm{a}-\mathrm{d}$ & $1.810 \mathrm{a}-\mathrm{c}$ & 5.164 a-d & $3.663 \mathrm{a}-\mathrm{d}$ \\
\hline 14 & H.12406 = BUC “S" / PVN “S" / /IAC-24 & $4.092 \mathrm{a}-\mathrm{d}$ & $1.652 \mathrm{a}-\mathrm{d}$ & $4.526 \mathrm{~cd}$ & $3.423 \mathrm{~b}-\mathrm{g}$ \\
\hline 15 & CHIL “S”/SUSHOE 10 & $3.313 \mathrm{~d}$ & $1.366 \mathrm{~b}-\mathrm{d}$ & $3.063 \mathrm{e}$ & $2.581 \mathrm{~h}$ \\
\hline 16 & PEA “S”/YMI 6 & $4.191 \mathrm{a}-\mathrm{c}$ & $1.701 \mathrm{a}-\mathrm{d}$ & $4.295 \mathrm{~d}$ & $3.396 \mathrm{c}-\mathrm{g}$ \\
\hline 17 & H.12058 = IAC-221/IAC-60 & $3.880 \mathrm{a}-\mathrm{d}$ & $1.642 \mathrm{a}-\mathrm{d}$ & $4.623 \mathrm{~b}-\mathrm{d}$ & $3.382 \mathrm{c}-\mathrm{g}$ \\
\hline 18 & H.11259 = 7C /IAC-21 & $3.781 \mathrm{a}-\mathrm{d}$ & $1.482 \mathrm{a}-\mathrm{d}$ & $4.241 \mathrm{~d}$ & $3.168 \mathrm{fg}$ \\
\hline 19 & IAC-289 & $4.615 \mathrm{a}$ & $1.807 \mathrm{a}-\mathrm{c}$ & 5.708 a & $4.043 \mathrm{a}$ \\
\hline \multirow[t]{2}{*}{20} & JCAM/EMU“S”//CHRC“S”/4/IAS20//WTE & & & & \\
\hline & *2/NAR/3/KVK/5/SHANGHAI 4 & $3.337 \mathrm{~b}-\mathrm{d}$ & $1.449 \mathrm{~b}-\mathrm{d}$ & $4.416 \mathrm{~d}$ & $3.201 \mathrm{e}-\mathrm{g}$ \\
\hline \multicolumn{2}{|c|}{ F (Genótipos) } & $5,48^{*}$ & $3,83^{*}$ & $9,61^{*}$ & $14,03^{*}$ \\
\hline \multicolumn{2}{|c|}{ F (Anos) } & $180,95^{*}$ & $53,01^{*}$ & 4,10 & $102,39 *$ \\
\hline \multicolumn{2}{|c|}{ F (Locais) } & - & - & - & $1.029,51^{*}$ \\
\hline \multicolumn{2}{|c|}{$F(G x A)$} & $5,09^{*}$ & $2,02^{*}$ & $5,19^{*}$ & $7,26^{*}$ \\
\hline \multicolumn{2}{|c|}{$\mathrm{F}(\mathrm{GxL})$} & - & - & - & $3,81^{*}$ \\
\hline \multicolumn{2}{|c|}{$F(G x A x L)$} & - & - & - & $3,39^{*}$ \\
\hline \multicolumn{2}{|c|}{ d.m.s. (Tukey a 5\%) } & 868 & 554 & 989 & 455 \\
\hline \multicolumn{2}{|c|}{ C.V.\% } & 12,30 & 19,48 & 11,37 & 13,29 \\
\hline
\end{tabular}

*Significativo ao nível de 5\%.

celas, compostas por seis concentraçoes de alumínio, e as subparcelas, pelos genótipos de trigo. Foram feitas duas repetições para cada solução-tratamento. $\mathrm{Na}$ análise dos dados considerou-se a média do com- primento da raiz primária central das dez plantas de cada genótipo, em 72 horas de crescimento, nas soluções nutritivas completas sem alumínio, que se seguiu a 48 horas de crescimento nas soluções de 
tratamento contendo seis diferentes concentrações de alumínio, conforme CAMARGo (1984) e Moore et al. (1976).

\section{RESULTADOS E DISCUSSÃO}

Os quadrados médios das análises individuais da variância da produção de grãos dos genótipos estudados em três locais paulistas, em 1996-97, mostraram-se significativos ao nível de 5\%, para genótipos, e não-significativos para repetições, em todos os experimentos.

Os quadrados médios da análise da variância conjunta das produções de grãos dos genótipos avaliados nos dois experimentos de Tatuí (1996-97), utilizandose irrigação por aspersão, mostraram-se significativos para anos, genótipos e para a interação genótipos $\mathrm{x}$ anos. Considerando a média dos ensaios de Tatuí (Quadro 2), verificou-se que o cultivar de trigo IAC289 e a linhagem L9, produzindo $4.615 \mathrm{~kg} \cdot \mathrm{ha}^{-1}$ foram os mais produtivos, diferindo, porém, somente das linhagens L1 (3.564 kg.ha $\left.{ }^{-1}\right)$, L4 (3.529 kg.ha $\left.{ }^{-1}\right)$, L5 (3.379 kg.ha $\left.{ }^{-1}\right)$, L7 (3.265 kg.ha $\left.{ }^{-1}\right)$, L15 (3.313 kg.ha $\left.{ }^{-1}\right)$ e L20 (3.337 kg.ha- ${ }^{-1}$ ). Nessas condições destacou-se, também pela produtividade, 'IAC-24' (4.514 kg.ha-1).

Os quadrados médios da análise de variância conjunta das produções médias de grãos, em quilogramas por hectare, dos genótipos dos ensaios de Cruzália, no período 1997-98, instalados em condição de sequeiro, mostraram efeitos significativos para genótipos, anos e para a interação genótipos $\mathrm{x}$ anos (Quadro 2). Observou-se, considerando conjuntamente esses dois ensaios, que a linhagem L9 $\left(2.010 \mathrm{~kg} \cdot \mathrm{ha}^{-1}\right)$ mostrou-se a mais produtiva, diferindo apenas das linhagens L2, L4, L6, L7, L8, L15 e L20, as menos produtivas. Nessas condições, destacaramse também pela produtividade as linhagens L11 (1.863 kg.ha- ${ }^{-1}$ ) e L12 (1.837 kg.ha-1).

Os quadrados médios da análise de variância conjunta das produções médias de grãos dos genótipos estudados nos ensaios de Ribeirão Preto (1996-97), em condição de irrigação por aspersão, apresentaram efeitos significativos para genótipos e para a interação genótipos $\mathrm{x}$ anos. As produções médias de grãos dos genótipos avaliados em Ribeirão Preto (1996-97) são apresentadas no quadro 2. Considerando-se conjuntamente esses dois ensaios observou-se que o cultivar de trigo IAC-289 (5.708 kg.ha $\left.{ }^{-1}\right)$ e a linhagem L11 $\left(5.603 \mathrm{~kg} \cdot \mathrm{ha}^{-1}\right)$ mostraram-se mais produtivos, diferindo somente das linhagens L1, L7, L14, L15, L16, L18 e L20, as quais exibiram as menores produtividades. O cultivar IAC-289 também diferiu da linhagem L17 (4.623 kg.ha- $\left.{ }^{-1}\right)$.
Avaliando em conjunto os seis experimentos, foram verificados, quanto à produção de grãos, efeitos significativos para genótipos, anos, locais e para as interações genótipos x anos, genótipos x locais e genótipos $x$ anos $x$ locais (Quadro 2). Pelo teste de Tukey, 'IAC-289' (4.043 kg.ha-1) e as linhagens L9 (4.021 kg.ha-1) e L11 (3.862 kg.ha ${ }^{-1}$ ) foram os mais produtivos. O cultivar IAC-289 e a linhagem L9 diferiram dos demais genótipos, com exceção de 'IAC-24' (3.645 kg.ha-1) e das linhagens L11 (3.862 kg.ha ${ }^{-1}$ ), L12 (3.831 kg.ha ${ }^{-1}$ ) e L14 (3.663 kg.ha $\left.{ }^{-1}\right)$.

Um aspecto a ser considerado nos resultados dos seis experimentos consiste no fato de que L9, provinda de cruzamento e seleções realizadas pelo Instituto Agronômico, foi a mais produtiva em Tatuí (condição de irrigação) e Cruzália (condição de sequeiro), indicando que um programa de melhoramento genético visando aumentar a produtividade dos genótipos de trigo, a partir de hibridações seguidas de seleções nas diferentes condições paulistas, teria grande probabilidade de sucesso.

As alturas médias das plantas, as porcentagens médias de acamamento e os ciclos médios da emergência à maturação dos genótipos estudados nos ensaios encontram-se no quadro 3.

'IAC-289' exibiu as plantas mais altas, diferindo dos demais genótipos pelo teste de Tukey ao nível de $5 \%$, à exceção do cultivar IAC-24 e das linhagens L 12, L14 e L18. Apresentou também a maior porcentagem de acamamento (14\%). As linhagens L2, L5, L6, L7 e L8 mostraram, ao mesmo tempo, as plantas mais baixas e menor porcentagem de plantas acamadas.

As linhagens L3, L8 e L15 foram precoces, com um ciclo médio da emergência à maturação entre $110 \mathrm{a}$ 120 dias. Os demais genótipos apresentaram um ciclo médio.

A linhagem L8, pelo porte baixo de planta associado à resistência ao acamamento e pelo ciclo precoce da emergência à maturação, representa germoplasma de grande interesse aos programas de melhoramento genético do trigo.

No quadro 4 são apresentados os dados médios de porcentagem de ferrugem-da-folha dos 20 genótipos de trigo avaliados nos experimentos instalados em Ribeirão Preto, Tatuí e Cruzália, nos anos de 1996 e 1997.

Houve baixa incidência da doença nos experimentos semeados em Ribeirão Preto e Cruzália, em 1996, mas pela alta intensidade ocorrida em Tatuí, em ambos os anos, e em 1997, tanto em Ribeirão Preto como em Cruzália, foi possível uma diferenciação de comportamento entre os genótipos estudados. Observando-se de forma geral o quadro 4, nota-se que os genótipos 1, 5, 13, 17 e 20 apresentaram baixos índices 
Quadro 3. Altura média de plantas, porcentagem média de acamamento e ciclo da emergência à maturação ${ }^{(1)}$ dos 20 genótipos de trigo avaliados nos experimentos instalados nas Estações Experimentais de Tatuí e de Ribeirão Preto, e na Fazenda Santa Lúcia, em Cruzália, em 1996 e 1997

\begin{tabular}{|c|c|c|c|}
\hline Genótipos & Altura & Acabamento & $\begin{array}{c}\text { Ciclo } \\
\text { Emerg.mat }\end{array}$ \\
\hline & $\mathrm{cm}$ & $\%$ & \\
\hline 01 & $70 \mathrm{ab}$ & 6 & Médio \\
\hline 02 & $48 \mathrm{~h}$ & 2 & Médio \\
\hline 03 & $58 \mathrm{~d}-\mathrm{f}$ & 3 & Precoce \\
\hline 04 & $57 e-g$ & 2 & Médio \\
\hline 05 & $51 \mathrm{f}-\mathrm{h}$ & 3 & Médio \\
\hline 06 & $50 \mathrm{gh}$ & 0 & Médio \\
\hline 07 & $50 \mathrm{gh}$ & 0 & Médio \\
\hline 08 & $53 f-h$ & 2 & Precoce \\
\hline 09 & $66 \mathrm{bc}$ & 8 & Médio \\
\hline 10 & $72 \mathrm{ab}$ & 12 & Médio \\
\hline 11 & $65 b-d$ & 3 & Médio \\
\hline 12 & $69 \mathrm{ab}$ & 6 & Médio \\
\hline 13 & $61 c-e$ & 3 & Médio \\
\hline 14 & $70 \mathrm{ab}$ & 7 & Médio \\
\hline 15 & $67 \mathrm{bc}$ & 4 & precoce \\
\hline 16 & $65 b-d$ & 9 & médio \\
\hline 17 & $65 b-d$ & 6 & médio \\
\hline 18 & $69 \mathrm{ab}$ & 5 & médio \\
\hline 19 & $76 a$ & 13 & médio \\
\hline 20 & $61 c-e$ & 2 & médio \\
\hline F(Genótipos) & $31,21^{*}$ & - & - \\
\hline F(Experimentos) & $134,67^{*}$ & - & - \\
\hline d.m.s. (Tukey a 5\%) & 8 & - & - \\
\hline C.V.\% & 5,87 & - & - \\
\hline
\end{tabular}

${ }^{(1)}$ Precoce $=110$ a 120 dias e médio $=120$ a 130 dias da emergência à maturação.

* significativo ao nível de 5\%.

de ferrugem nas três localidades, enquanto os genótipos 15 e 16 não revelaram bom comportamento frente à ferrugem-da-folha apenas em 1997, em Cruzália, possivelmente devido à ocorrência de raça diferente ou de mudança de prevalência de raça do patógeno. Os genótipos 1, 17 e 20, que revelaram características de resistência, dada pela baixa severidade da doença em todos os ensaios, poderão ser utilizados como fontes de resistência em programa de melhoramento.
Quadro 4. Graus médios de infecção ${ }^{(1)}$ de ferrugem-da-folha dos 20 genótipos de trigo avaliados nos experimentos instalados nas Estações Experimentais de Tatuí e de Ribeirão Preto, e na Fazenda Santa Lúcia, em Cruzália, em 1996 e 1997

\begin{tabular}{|c|c|c|c|c|c|c|}
\hline \multirow{3}{*}{ Genótipos } & \multicolumn{6}{|c|}{ Ferrugem-da-folha } \\
\hline & \multicolumn{2}{|c|}{ Ribeirão Preto } & \multicolumn{2}{|c|}{ Tatuí } & \multicolumn{2}{|c|}{ Cruzália } \\
\hline & 1996 & 1997 & 1996 & 1997 & 1996 & 1997 \\
\hline 01 & 0 & $5 S$ & $3 S$ & 0 & $\mathrm{t} S$ & 0 \\
\hline 02 & $5 S$ & $33 S$ & $8 \mathrm{~S}$ & $17 \mathrm{~S}$ & $5 S$ & $20 \mathrm{~S}$ \\
\hline 03 & $\mathrm{tS}$ & $23 \mathrm{~S}$ & $20 \mathrm{~S}$ & $35 \mathrm{~S}$ & $3 S$ & $20 S$ \\
\hline 04 & $3 S$ & $15 \mathrm{~S}$ & $51 S$ & $33 \mathrm{~S}$ & $5 S$ & $20 \mathrm{~S}$ \\
\hline 05 & 0 & $3 S$ & $8 \mathrm{~S}$ & $4 S$ & $\mathrm{t} S$ & $20 \mathrm{~S}$ \\
\hline 06 & 0 & $50 \mathrm{~S}$ & $31 S$ & $40 \mathrm{~S}$ & $3 S$ & $30 S$ \\
\hline 07 & tS & $3 S$ & $18 \mathrm{~S}$ & $32 \mathrm{~S}$ & 0 & $40 \mathrm{~S}$ \\
\hline 08 & $3 S$ & $18 \mathrm{~S}$ & $18 \mathrm{~S}$ & $32 \mathrm{~S}$ & $5 S$ & $20 \mathrm{~S}$ \\
\hline 09 & 0 & $10 \mathrm{~S}$ & $28 \mathrm{~S}$ & $22 \mathrm{~S}$ & $\mathrm{tS}$ & $20 S$ \\
\hline 10 & 0 & $6 S$ & $20 S$ & $22 \mathrm{~S}$ & 0 & $30 S$ \\
\hline 11 & 0 & $15 \mathrm{~S}$ & $33 S$ & $15 \mathrm{~S}$ & $3 S$ & $30 S$ \\
\hline 12 & $\mathrm{tS}$ & $28 \mathrm{~S}$ & $33 \mathrm{~S}$ & $30 \mathrm{~S}$ & $5 S$ & $30 S$ \\
\hline 13 & 0 & $5 S$ & $20 \mathrm{~S}$ & $7 \mathrm{~S}$ & 0 & $10 \mathrm{~S}$ \\
\hline 14 & 0 & $25 \mathrm{~S}$ & $14 \mathrm{~S}$ & $18 \mathrm{~S}$ & $3 S$ & $20 \mathrm{~S}$ \\
\hline 15 & 0 & $\mathrm{t} S$ & tS & $5 S$ & $5 S$ & $30 S$ \\
\hline 16 & $\mathrm{t} S$ & $6 S$ & $8 \mathrm{~S}$ & $13 S$ & $3 S$ & $30 S$ \\
\hline 17 & 0 & $\mathrm{t} S$ & $8 \mathrm{~S}$ & $7 \mathrm{~S}$ & 0 & $10 \mathrm{~S}$ \\
\hline 18 & 0 & $5 S$ & $31 \mathrm{~S}$ & $18 \mathrm{~S}$ & 0 & $30 S$ \\
\hline 19 & 0 & $40 \mathrm{~S}$ & $16 \mathrm{~S}$ & $10 \mathrm{~S}$ & 0 & $20 S$ \\
\hline 20 & 0 & $3 S$ & $6 \mathrm{~S}$ & $7 \mathrm{~S}$ & $3 S$ & $5 S$ \\
\hline
\end{tabular}

${ }^{(1)}$ Avaliação da ferrugem-da-folha segundo Mehta (1978); $\mathrm{t}=$ traço (apenas algumas pústulas); $\mathrm{S}=$ reação de suscetibilidade.

Quanto à mancha foliar, sua ocorrência em Ribeirão Preto nos dois anos, e em Cruzália no ano de 1996, não permitiu a realização de avaliação devido à baixa severidade da doença. Por outro lado, sua ocorrência caracterizou-se por apresentar maior severidade nos experimentos instalados em Tatuí, em ambos os anos, e em Cruzália, no ano de 1997. Contudo, não foi possível diferenciar, de forma significativa, os genótipos, quanto à resistência a essa doença. Pelos resultados apresentados no quadro 5 , verifica-se que todos os genótipos foram suscetíveis, não permitindo estabelecer diferenças de níveis de resistência entre os mesmos, classificando-se apenas o cultivar IAC-289 como moderadamente resistente, em Tatuí, com índices de $23 \%$ e $24 \%$ de área foliar afetada. Em Cruzália, porém, em 1997, esse cultivar comportou-se como 
suscetível. Também em vários outros trabalhos (CAMARGO et al., 1994; 1995a,b) já foi confirmada a dificuldade de encontrar genótipos com boa resistência a Bipolaris sorokiniana.

Com relação ao oídio ocorrido em Ribeirão Preto e Tatuí, neste último local com alta severidade, alguns genótipos mais suscetíveis alcançaram índices superiores a 40\%, em 1996. Comparando-se os graus médios de infecção de oídio, observa-se que houve ampla variação entre os genótipos (Quadro 6). Os resultados apresentados indicam que as condições ambientais foram favoráveis ao desenvolvimento do oídio. De modo geral, os cultivares e linhagens 1, 2, 6, $7,8,9,11,12,14,15,16$ e 18 apresentaram-se como mais suscetíveis que os demais. Por outro lado, a

Quadro 5. Graus médios de infecção ${ }^{(1)}$ de mancha foliar dos 20 genótipos de trigo avaliados nos experimentos instalados nas Estações Experimentais de Tatuí e de Ribeirão Preto, e na Fazenda Santa Lúcia, em Cruzália, em 1996 e 1997

\begin{tabular}{|c|c|c|c|c|c|c|}
\hline \multirow{3}{*}{ Genótipos } & \multicolumn{6}{|c|}{ Mancha foliar } \\
\hline & \multicolumn{2}{|c|}{ Ribeirão Preto } & \multicolumn{2}{|c|}{ Tatuí } & \multicolumn{2}{|c|}{ Cruzália } \\
\hline & 1996 & 1997 & 1996 & 1997 & 1996 & 1997 \\
\hline 01 & - & - & 35 & 40 & 5 & 20 \\
\hline 02 & - & - & 42 & 40 & 5 & 40 \\
\hline 03 & - & - & 32 & 33 & 8 & 40 \\
\hline 04 & - & - & 35 & 33 & 5 & 40 \\
\hline 05 & - & - & 40 & 33 & 8 & 40 \\
\hline 06 & 1 & - & 40 & 40 & 8 & 40 \\
\hline 07 & - & - & 37 & 33 & 5 & 40 \\
\hline 08 & - & - & 35 & 33 & 8 & 40 \\
\hline 09 & - & - & 29 & 30 & 5 & 30 \\
\hline 10 & 1 & - & 29 & 30 & 8 & 30 \\
\hline 11 & - & - & 29 & 33 & 8 & 30 \\
\hline 12 & - & - & 27 & 33 & 5 & 30 \\
\hline 13 & - & - & 32 & 37 & 8 & 30 \\
\hline 14 & - & - & 32 & 30 & 5 & 40 \\
\hline 15 & 5 & - & 24 & 30 & 8 & 30 \\
\hline 16 & - & - & 35 & 30 & 5 & 30 \\
\hline 17 & - & - & 31 & 30 & 8 & 30 \\
\hline 18 & - & - & 45 & 40 & 8 & 40 \\
\hline 19 & - & - & 24 & 23 & 5 & 30 \\
\hline 20 & - & - & 32 & 30 & 5 & 30 \\
\hline
\end{tabular}

${ }^{(1)}$ Avaliação da mancha-da-folha, segundo Mehta (1978): $0=$ imune; $1 \%$ a $5 \%$ de área infectada $=$ resistente; $6 \%$ a $25 \%$ = moderadamente resistente; $26 \%$ a $50 \%$ = suscetível, e $51 \%$ a $99 \%$ = altamente suscetível; $\mathrm{t}=$ traço (apenas algumas pústulas). menor severidade de sintomas foi observada no cultivar IAC-24, confirmando resultados anteriores relatados por Felício et al. (1988) e CAMARGo et al. (1995c). Os genótipos 4, 5, 10, 17 e 20 apresentaram-se resistentes ao oídio em, pelo menos, três ensaios.

Através da análise dos resultados observa-se que houve variação nos graus médios de infecção dos três patógenos considerados em diversos genótipos, nos três locais e nos dois anos estudados, causando alteração na reação varietal para cada patógeno (Quadros 4 a 6). As causas de tais variações podem ter sido pequenas oscilações ambientais de umidade e temperatura, as quais constituem importantes fatores que influenciam o desenvolvimento das doenças. Outro fato que pode ter contribuído para tais variações é a

Quadro 6. Graus médios de infecção ${ }^{(1)}$ de oídio dos 20 genótipos de trigo avaliados nos experimentos instalados nas Estações Experimentais de Tatuí e de Ribeirão Preto, e na Fazenda Santa Lúcia, em Cruzália, em 1996 e 1997

\begin{tabular}{|c|c|c|c|c|c|c|}
\hline \multirow{3}{*}{ Genótipos } & \multicolumn{6}{|c|}{ Oídio } \\
\hline & \multicolumn{2}{|c|}{ Ribeirão Preto } & \multicolumn{2}{|c|}{ Tatuí } & \multicolumn{2}{|c|}{ Cruzália } \\
\hline & 1996 & 1997 & 1996 & 1997 & 1996 & 1997 \\
\hline 01 & 5 & 18 & 35 & 13 & 5 & - \\
\hline 02 & 8 & 15 & 42 & 15 & 0 & - \\
\hline 03 & 10 & 8 & 32 & 2 & 0 & - \\
\hline 04 & $\mathrm{t}$ & 8 & 35 & 2 & $\mathrm{t}$ & - \\
\hline 05 & 0 & 5 & 40 & 2 & 3 & - \\
\hline 06 & 3 & 30 & 40 & 15 & 0 & - \\
\hline 07 & 3 & 50 & 37 & 37 & 3 & - \\
\hline 08 & 5 & 10 & 35 & 14 & 3 & - \\
\hline 09 & 8 & 25 & 29 & 10 & $\mathrm{t}$ & - \\
\hline 10 & $\mathrm{t}$ & 5 & 29 & 5 & $\mathrm{t}$ & - \\
\hline 11 & 10 & 15 & 29 & 14 & 0 & - \\
\hline 12 & 15 & 10 & 27 & 17 & 0 & - \\
\hline 13 & 3 & 8 & 32 & 23 & 3 & - \\
\hline 14 & 3 & 30 & 32 & 27 & 3 & - \\
\hline 15 & 8 & 33 & 24 & 12 & 3 & - \\
\hline 16 & 23 & 18 & 35 & 37 & 3 & - \\
\hline 17 & $t$ & 3 & 31 & 15 & 0 & - \\
\hline 18 & $\mathrm{t}$ & 18 & 45 & 15 & 0 & - \\
\hline 19 & $\mathrm{t}$ & 15 & 24 & 8 & 0 & - \\
\hline 20 & 3 & 6 & 32 & 8 & 0 & - \\
\hline
\end{tabular}

${ }^{(1)}$ Avaliação de oídio segundo Mehta (1978): 0 = imune; $1 \%$ a $5 \%$ de área infectada $=$ resistente $; \%$ a $25 \%=$ moderadamente resistente; $26 \%$ a $50 \%$ = suscetível, e $51 \%$ a $99 \%$ = altamente suscetível; $\mathrm{t}=$ traço (apenas algumas pústulas). 
mudança de prevalência de raças do patógeno, em função do local ou ano considerado.

Os quadrados médios das análises conjuntas da variância para comprimento da espiga, número de espiguetas por espiga, número de grãos por espiga e por espigueta, e massa de cem grãos dos genótipos dos ensaios de Tatuí e de Ribeirão Preto, em 1996 e 1997, mostraram, para todas as características consideradas, efeitos significativos para genótipos, para experimentos, e interação genótipos $\mathrm{x}$ experimentos (Quadro 7).

A linhagem L7 apresentou, ao mesmo tempo, as espigas mais compridas e o maior número de espiguetas por espiga, diferindo, pelo teste de Tukey ao nível de $5 \%$, de todos os genótipos avaliados, com exceção da linhagem L8 e do cultivar IAC-289, para comprimento da espiga, e das linhagens L3 e L12 para número de espiguetas por espiga (Quadro 7). A linhagem L7 constitui germoplasma de interesse ao melhoramento do trigo como fonte dessas características, em programas de cruzamentos.

O cultivar IAC-289 exibiu o maior número de grãos por espiga, não diferindo significativamente ao nível de $5 \%$ pelo teste de Tukey dos demais genótipos, com exceção do cultivar IAC-24 e das linhagens L2, L15 e L18 (Quadro 7).

Em relação ao número de grãos por espigueta, a linhagem L16 apresentou o maior valor, somente diferindo do cultivar IAC-24 e da linhagem L15, que apresentaram pequena fertilidade da espiga, representada por menor número de grãos por espigueta (Quadro 7).

L11 apresentou os grãos mais pesados, não diferindo apenas de L12 (Quadro 7), constituindo-se em germoplasma de grande interesse aos programas de melhoramento de trigo, quando essa característica for desejada.

Encontram-se no quadro 8 o comprimento médio das raízes dos 20 genótipos de trigo avaliados, além dos cultivares-controle BH-1146 (tolerante) e Anahuac (sensível), após 72 horas de crescimento em solução normal (sem alumínio), que se seguiu a um crescimento em solução-tratamento contendo seis diferentes concentrações de alumínio, referente à média das duas repetições.

Considerando $2 \mathrm{mg} . \mathrm{L}^{-1}$ de $\mathrm{Al}^{3+}$ (Quadro 8), verificou-se que as linhagens L15 e L20 e o cultivar-controle Anahuac foram sensíveis a essa concentração de alumínio, ou seja, revelaram paralisação irreversível do crescimento da raiz primária central após tratamento com $2 \mathrm{mg} . \mathrm{L}^{-1}$ de $\mathrm{Al}^{3+}$. Os demais genótipos mostraram-se tolerantes, isto é, exibiram algum crescimento radicular após tratamentos em soluções contendo essa concentração de alumínio. $\mathrm{O}$ mesmo
Quadro 7. Dados médios referentes ao comprimento da espiga, número de espiguetas por espiga, número de grãos por espiga e por espigueta e massa de cem grãos dos 20 genótipos de trigo avaliados nos experimentos instalados nas Estações Experimentais de Tatuí e de Ribeirão Preto, em 1996 e 1997

\begin{tabular}{|c|c|c|c|c|c|}
\hline Genótipos & $\begin{array}{l}\text { Compr. } \\
\text { espiga }\end{array}$ & $\begin{array}{c}\text { Espigueta } \\
\text { / espiga }\end{array}$ & $\begin{array}{c}\text { Grãos / } \\
\text { espiga }\end{array}$ & $\begin{array}{l}\text { Grãos / } \\
\text { espigueta }\end{array}$ & $\begin{array}{l}\text { Massa de } \\
\text { cem } \\
\text { grãos }\end{array}$ \\
\hline & $\mathrm{cm}$ & 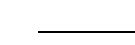 & n ${ }^{o}$ & & $\mathrm{~g}$ \\
\hline 01 & 7,7 b-f & $16,3 \mathrm{~g}$ & 38,9 a-d & $2,43 \mathrm{a}-\mathrm{c}$ & 4,32 b-d \\
\hline 02 & $7,4 \mathrm{~d}-\mathrm{g}$ & $17,4 \mathrm{~d}-\mathrm{g}$ & 37,2 b-d & 2,15 a-c & 3,52 h-k \\
\hline 03 & $8,3 \mathrm{bc}$ & $19,7 \mathrm{ab}$ & 44,5 a-c & $2,23 \mathrm{a}-\mathrm{c}$ & 3,50 h-k \\
\hline 04 & 7,9 b-e & $18,9 \mathrm{~b}-\mathrm{d}$ & $47,7 \mathrm{ab}$ & $2,49 \mathrm{a}-\mathrm{c}$ & $3,38 \mathrm{jk}$ \\
\hline 05 & $7,7 \mathrm{~b}-\mathrm{f}$ & $19,1 \mathrm{bc}$ & 45,4 a-c & 2,36 a-c & 3,39 i-k \\
\hline 06 & 7,8 b-f & $16,9 \mathrm{fg}$ & 45,6 a-c & $2,68 \mathrm{a}$ & $3,54 \mathrm{~g}-\mathrm{k}$ \\
\hline 07 & $9,2 \mathrm{a}$ & $21,1 \mathrm{a}$ & $46,5 \mathrm{ab}$ & $2,18 \mathrm{a}-\mathrm{c}$ & $3,26 \mathrm{k}$ \\
\hline 08 & $8,4 \mathrm{ab}$ & $19,2 \mathrm{bc}$ & $46,9 \mathrm{ab}$ & $2,42 \mathrm{a}-\mathrm{c}$ & $4,10 \mathrm{c}-\mathrm{f}$ \\
\hline 09 & 7,5 c-g & $17,2 \mathrm{e}-\mathrm{g}$ & $42,4 \mathrm{a}-\mathrm{d}$ & 2,46 a-c & $4,13 \mathrm{c}-\mathrm{f}$ \\
\hline 10 & $7,4 \mathrm{~d}-\mathrm{g}$ & $18,1 \mathrm{c}-\mathrm{f}$ & $37,8 \mathrm{~b}-\mathrm{d}$ & $2,06 \mathrm{bc}$ & $3,79 e-j$ \\
\hline 11 & $7,5 \mathrm{c}-\mathrm{g}$ & $16,8 \mathrm{fg}$ & $41,5 \mathrm{a}-\mathrm{d}$ & $2,45 \mathrm{a}-\mathrm{c}$ & $4,98 \mathrm{a}$ \\
\hline 12 & $8,2 \mathrm{~b}-\mathrm{d}$ & $19,7 \mathrm{ab}$ & $43,1 \mathrm{a}-\mathrm{c}$ & $2,20 \mathrm{a}-\mathrm{c}$ & $4,74 \mathrm{ab}$ \\
\hline 13 & $7,3 \mathrm{e}-\mathrm{g}$ & $16,6 \mathrm{fg}$ & 38,8 a-d & $2,34 \mathrm{a}-\mathrm{c}$ & $3,67 \mathrm{f}-\mathrm{k}$ \\
\hline 14 & $7,5 \mathrm{c}-\mathrm{g}$ & $17,1 \mathrm{e}-\mathrm{g}$ & $42,1 \mathrm{a}-\mathrm{d}$ & $2,46 \mathrm{a}-\mathrm{c}$ & $3,95 \mathrm{~d}-\mathrm{h}$ \\
\hline 15 & $6,7 \mathrm{gh}$ & $16,5 \mathrm{~g}$ & $32,2 \mathrm{~d}$ & $1,94 \mathrm{c}$ & $4,45 \mathrm{bc}$ \\
\hline 16 & $6,3 \mathrm{~h}$ & 17,3 e-g & $46,7 \mathrm{ab}$ & $2,67 a$ & $3,87 \mathrm{~d}-\mathrm{i}$ \\
\hline 17 & $7,3 \mathrm{e}-\mathrm{g}$ & $17,1 \mathrm{e}-\mathrm{g}$ & $42,8 \mathrm{a}-\mathrm{c}$ & $2,48 \mathrm{a}-\mathrm{c}$ & $4,00 \mathrm{c}-\mathrm{g}$ \\
\hline 18 & $7,0 \mathrm{f}-\mathrm{h}$ & $15,9 \mathrm{~g}$ & $35,4 \mathrm{~cd}$ & 2,17 a-c & 4,27 b-e \\
\hline 19 & $8,4 \mathrm{ab}$ & 19,4 bc & $49,2 \mathrm{a}$ & $2,54 \mathrm{ab}$ & $3,77 \mathrm{f}-\mathrm{j}$ \\
\hline 20 & $6,8 \mathrm{gh}$ & 18,6 b-e & $41,9 \mathrm{a}-\mathrm{d}$ & 2,26 a-c & 3,50 h-k \\
\hline $\begin{array}{l}\text { F (Genó- } \\
\text { tipos) }\end{array}$ & $15,13^{*}$ & $23,71^{*}$ & $4,92^{*}$ & $3,04^{*}$ & $25,20^{*}$ \\
\hline $\begin{array}{l}\mathrm{F} \text { (Expe- } \\
\text { rimentos) }\end{array}$ & $17,40^{*}$ & $27,83^{*}$ & $10,28^{*}$ & $5,14^{*}$ & $37,45^{*}$ \\
\hline$F(G \times E)$ & $1,67^{*}$ & $2,08^{*}$ & $2,37^{*}$ & $3,34^{*}$ & $3,60^{*}$ \\
\hline $\begin{array}{l}\text { d.m.s. } \\
\text { (Tukey a } \\
5 \% \text { ) }\end{array}$ & 0,9 & 1,6 & 10,6 & 0,59 & 0,49 \\
\hline C.V. \% & 7,07 & 4,46 & 12,37 & 10,44 & 5,06 \\
\hline
\end{tabular}

*Significativo ao nível de 5\%.

resultado foi observado quando foram adicionados 4 e $6 \mathrm{mg} . \mathrm{L}^{-1}$ nas soluções de tratamento.

O genótipo 16 foi considerado sensível à concentração de $8 \mathrm{mg} \cdot \mathrm{L}^{-1}$ de $\mathrm{Al}^{3+}$ e os genótipos 5 e 19, sensíveis a $10 \mathrm{mg} . \mathrm{L}^{-1}$ de $\mathrm{Al}^{3+}$. Os genótipos $1,2,3,4$, $6,7,8,9,10,11,12,13,14,17,18$ e o cultivar BH-1146 
Quadro 8. Comprimento médio das raízes dos 20 genótipos de trigo avaliados após 72 horas de crescimento em solução normal, que se seguiu a um crescimento em solução tratamento contendo seis diferentes concentrações de alumínio

\begin{tabular}{|c|c|c|c|c|c|c|}
\hline \multirow[t]{2}{*}{ Genótipos } & \multicolumn{6}{|c|}{ Concentrações de alumínio $\left(\mathrm{mg} . \mathrm{L}^{-1}\right)$} \\
\hline & 0 & 2 & 4 & 6 & 8 & 10 \\
\hline & & & $-n$ & $n$ & 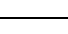 & 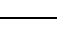 \\
\hline 01 & 89,9 & 50,9 & 23,6 & 20,3 & 9,0 & 1,1 \\
\hline 02 & 104,5 & 50,1 & 33,1 & 28,6 & 11,3 & 0,7 \\
\hline 03 & 82,7 & 46,7 & 39,9 & 32,6 & 19,1 & 10,5 \\
\hline 04 & 82,6 & 42,8 & 16,8 & 5,1 & 2,8 & 1,5 \\
\hline 05 & 88,9 & 32,8 & 18,1 & 15,9 & 2,3 & 0,0 \\
\hline 06 & 79,1 & 49,2 & 43,8 & 32,9 & 24,1 & 24,3 \\
\hline 07 & 105,6 & 51,3 & 31,0 & 12,9 & 3,9 & 0,6 \\
\hline 08 & 105,1 & 36,8 & 39,1 & 18,2 & 12,9 & 1,4 \\
\hline 09 & 96,4 & 61,9 & 26,6 & 8,9 & 5,9 & 7,0 \\
\hline 10 & 95,4 & 52,5 & 43,3 & 24,0 & 25,4 & 17,6 \\
\hline 11 & 93,1 & 30,3 & 13,8 & 10,5 & 5,9 & 1,9 \\
\hline 12 & 79,2 & 38,3 & 31,5 & 11,6 & 5,6 & 1,9 \\
\hline 13 & 97,8 & 45,3 & 20,5 & 8,4 & 3,9 & 0,1 \\
\hline 14 & 113,4 & 63,9 & 53,7 & 39,4 & 31,8 & 13,7 \\
\hline 15 & 90,2 & 0,0 & 0,0 & 0,0 & 0,0 & 0,0 \\
\hline 16 & 95,2 & 37,1 & 9,3 & 3,8 & 0,0 & 0,0 \\
\hline 17 & 86,9 & 49,7 & 34,3 & 20,3 & 13,0 & 5,6 \\
\hline 18 & 102,0 & 42,4 & 27,1 & 14,5 & 10,5 & 7,5 \\
\hline 19 & 91,9 & 37,9 & 15,7 & 2,9 & 0,2 & 0,0 \\
\hline 20 & 95,9 & 0,0 & 0,0 & 0,0 & 0,0 & 0,0 \\
\hline BH-1146 & 98,5 & 84,1 & 68,7 & 56,0 & 45,6 & 38,4 \\
\hline Anahuac & 98,8 & 0,0 & 0,0 & 0,0 & 0,0 & 0,0 \\
\hline
\end{tabular}

mostraram tolerância à toxicidade de alumínio, mesmo quando foram adicionados $10 \mathrm{mg} .{ }^{\mathrm{L}-1} \mathrm{de} \mathrm{Al}^{3+}$ nas soluções de tratamento. Os genótipos 3, 6 e 14 e os cultivares BH-1146 e IAC-24 apresentaram tolerância elevada, exibindo o maior crescimento das raízes, após permanecerem 48 horas em soluções contendo $10 \mathrm{mg} . \mathrm{L}^{-1}$ de $\mathrm{Al}^{3+}$.

\section{CONCLUSÕES}

1. A linhagem L9 e o cultivar IAC-289 destacaramse quanto à produção de grãos, considerando a média dos seis experimentos, não diferindo do cultivar IAC24 e das linhagens L11, L12 e L14.
2. A linhagem L8 exibiu porte baixo associado à resistência ao acamamento e ciclo precoce da emergência à maturação.

3. As linhagens L1, L17 e L20 revelaram características de resistência à ferrugem-da-folha, devido à baixa severidade da doença em todos os ensaios considerados.

4. Todos os genótipos mostraram-se suscetíveis aos agentes causais das manchas foliares e de oídio, à exceção do cultivar IAC-24, que apresentou menor severidade de sintomas em relação ao oídio.

5. A linhagem L7 produziu espigas mais compridas e maior número de espiguetas por espiga; o cultivar IAC-289, maior número de grãos por espiga; a linhagem L 16, maior número de grãos por espigueta, e a linhagem L11, grãos mais pesados.

6. Todos os genótipos avaliados mostraram-se tolerantes à toxicidade de $\mathrm{Al}^{3+}$, em condições de solução nutritiva, com exceção do cultivar-controle Anahuac e das linhagens L15 e L20, que exibiram elevada sensibilidade.

\section{REFERÊNCIAS BIBLIOGRÁFICAS}

CAMARGO, C.E. de O. Melhoramento do trigo: VI. Hereditariedade da tolerância a três concentrações de alumínio em solução nutritiva. Bragantia, Campinas, v.43, n.2, p.279-291, 1984.

CAMARGO, C.E. de O. Melhoramento genético do trigo para irrigação de inverno nas condições do Estado de São Paulo. In: SIMPÓSIO SOBRE MANEJO DE ÁGUA NA AGRICULTURA, 1987, Campinas. Resumos... Campinas: Fundação Cargill, 1987. p.134-174.

CAMARGO, C.E. de O. Trigo. In: FURLANI, A.M.C.; VIÉGAS, G.P. (Eds). O melhoramento de plantas no Instituto Agronômico. Campinas: Instituto Agronômico, 1993. p 433-488.

CAMARGO, C.E. de O.; FELÍCIO, J.C.; FERREIRA-FILHO, A.W.P. Variedades de trigo para o Estado de São Paulo. Campinas: Instituto Agronômico, 1996. 20p. (Boletim Técnico, 163)

CAMARGO, C.E. de O.; FELÍCIO, J.C.; FERREIRA-FILHO, A.W.P.; FREITAS, J.G. de; BARROS, B. deC.; CASTRO, J.L.; SABINO, J.C.; ROCHA-JUNIOR, L. S. Melhoramento de trigo: XXI. Avaliação de linhagens em diferentes regiões paulistas. Bragantia, Campinas, v. 48, n.1.p.53-71, 1989.

CAMARGO, C.E. de O.; FELÍCIO, J.C.; FERREIRA-FILHO, A.W.P.; BARROS, B. de C.; FREITAS, J.G. de; PETTINELLI-JUNIOR, A.; GALLO, P.B.; KANTHACK, R.A.D. Melhoramento de trigo: XXV. Avaliação de genótipos oriundos de populações híbridas introduzidas de Oregon (EUA) no Estado de São Paulo. Bragantia, Campinas, v.50, n.2, p.225-246, 1991. 
CAMARGO, C.E. de O.; FELÍCIO, J.C. ; FERREIRA-FILHO, A.W.P.; BARROS, B. de C.; SABINO, J.C. Melhoramento do trigo: comportamento de novas linhagens em diferentes regiões do Estado de São Paulo. Arquivos do Instituto Biológico, São Paulo, v.61, n.1/2, p.16-27, 1994.

CAMARGO, C.E. de O.; FELÍCIO, J.C.; TULMANN NETO, A.; FERREIRA-FILHO, A.W.P.; PETTINELLI-JUNIOR, A.; CASTRO, J.L. Melhoramento do trigo: XXVIII. Novos genótipos obtidos por seleções em população segregante interespecífica submetida à irradiação gama. Bragantia, Campinas, v.54, n.1, p.51-65, 1995a.

CAMARGO, C.E. de O.; FELÍCIO, J.C.; FERREIRA-FILHO, A.W.P.; FREITAS, J.G. de; RAMOS, V.J.; KANTHACK, R.A.D.; CASTRO, J.L. de. Melhoramento do trigo: XXX. Avaliação de linhagens com tolerância à toxicidade de alumínio, manganês e ferro em condição de campo. Bragantia, Campinas, v.54, n.1.p.81-93, 1995b.

CAMARGO, C.E. de O.; FELÍCIO, J.C.; FERREIRA-FILHO, A.W.P.; GALLO, P.B.; PETTINELLI-JUNIOR, A.; SANTOS, R.R dos; SABINO, J.C. Melhoramento do trigo: XXIX. Avaliação de linhagens da espécie Triticum durum L. no Estado de São Paulo. Bragantia, Campinas, v.54, n.1, p.67-79, 1995c.

FELÍCIO, J.C.; CAMARGO, C.E. de O.; BARROS, B. de C.; FERREIRA-FILHO, A.W.P.; GALLO, P.B.; RAMOS, V.J.; VITTI, P. IAC-60 Centenário e IAC-162 Tuiuiu: cultivares de trigo para sequeiro e irrigado no Estado deSão Paulo. Bragantia, Campinas, v.50, n.2, p.291-309, 1991.

FELÍCIO, J.C.; CAMARGO, C.E. de O.; FERREIRA FILHO, A.W.P.;FREITAS, J.G. de; VITTI, P. Tocantins (IAC 23) e Tucuruí (IAC 24): novos cultivares de trigo. Bragantia, Campinas, v.47, n.1, p.93-107, 1988.

FELÍCIO, J.C.; CAMARGO, C.E. de O.; MAGNO, C. de P.R. dos S.; PEREIRA, J.C.V.N.A.; PETTINELLI-JUNIOR, A. Avaliação agronômica e de qualidade tecnológica de genótipos de trigo com irrigação por aspersão no Estado de São Paulo. Bragantia, v.55, n.1, p.147-156, 1996.

FELÍCIO, J.C.; CAMARGO, C.E. de O.; VITTI, P.; CAMPAGNOLLI, D.M.F. Comportamento agronômico e avaliação tecnológica dos cultivares de trigo: IAC-120 (Curumi), IAC-286 (Takaoka) e IAC-289 (Marruá) para o Estado de São Paulo. Bragantia, Campinas, v.53, n.2, p.191-208, 1994a.

FELÍCIO, J.C.; CAMARGO, C.E. de O.; VITTI, P.; PEREIRA, J.C.V.N.A. Origem e avaliação de trigo: Tapajós (IAC72), Anhumas (IAC-227) e Yaco (IAC-287) para o Estado de São Paulo. Bragantia, Campinas, v.53, n.2, p.219-236, 1994b.

KOHLI, M.M.; CAMARGO, C.E. de O.; FRANCO, F.A. An analysis of the progress made in Brazil in breeding wheat for acid soils. In: TANNER, D.G. (Ed). Developing sustainable wheat production systems: the eighth regional wheat workshop for Eastern, Central and Southern Africa. Addis Abeba: CIMMYT, 1994. p.7898.

MEHTA, Y.R. Doenças do trigo e seu controle. São Paulo: Agronômica Ceres, 1978. 190p. (Ceres, 20)

MOORE, D.P.; KRONSTAD, W.E.; METZGER, R. J. Screening wheat for aluminum tolerance. In: WORKSHOP ON PLANT ADAPTATION TO MINERAL STRESS IN PROBLEM SOILS, 1976, Beltsville. Proceedings... Ithaca: Cornell University, 1976. p.287-295 